\title{
Adaptabilidade e estabilidade de genótipos de soja por meio de métodos uni e multivariado
}

\section{Adaptability and stability of soybean genotypes using univariate and multivariate methods}

\author{
Hélio Bandeira Barros ${ }^{1}$, Tuneo Sediyama ${ }^{2}$, Aurélio Vaz de Melo ${ }^{1}$, Rodrigo Ribeiro Fidelis ${ }^{1}$ \\ e Aristóteles Capone ${ }^{1^{*}}$
}

${ }^{1}$ Departamento de Agronomia; Universidade Federal do Tocantins; 77402-970; Gurupi - TO - Brasil.

${ }^{2}$ Departamento de Agronomia; Universidade Federal de Viçosa; 36570-000; Viçosa - MG - Brasil.

\begin{abstract}
This work aimed to evaluate the productivity, stability and adaptability of 29 soy genotypes [Glycine max (L.) Merr.] in six environments at the state of Mato Grosso. The experiments were laid out in a complete randomized blocks scheme with four replicates. The tests were carried out at 2004/05 agricultural year at the municipalities of Rondonópolis, Campo Verde, Nova Brasilândia and Vera. The stability and adaptability were evaluated according to the methods developed by Annicchiarico (1992) and Centroid method (Rocha et al., 2005). The mean yielding was $3054 \mathrm{~kg} \mathrm{ha}^{-1}$ (Rondonópolis III) to $3746 \mathrm{~kg} \mathrm{ha}^{-1}$ (Nova Brasilândia) with general mean among environments equal to $3292 \mathrm{~kg} \mathrm{ha}^{-1}$. The lines SL 1831, SL 1923, SL 627, SL 2280 and SL310 were classified by having the highest yielding, adaptability and stability according to the methodologies developed by Annicchiarico and Centroid, which indicate a consistency among the methodologies used. The lines SL 1831, SL 627, SL 2280 and SL310 were recommended for a wide range of environmental conditions and the line SL 818 was recommended to specific conditions of favorable environments.
\end{abstract}

Key-words: Glycine max, productivity, stability, adaptability, uni-multivariate

\section{INTRODUÇÃO}

Para a produtividade de grãos, o fenótipo de soja a ser selecionado depende do genótipo, do ambiente e da interação do genótipo $\mathrm{x}$ ambiente. Essa interação ocorre devido à inconsistência do desempenho dos genótipos nos vários ambientes, refletindo nas diferentes respostas dos genótipos às mudanças ambientais. Considerando as inúmeras variações ambientais em que a soja é comumente submetida, é esperado que a interação genótipos $\mathrm{x}$ ambiente assuma papel fundamental na manifestação fenotípica, devendo, portanto, ser estimada e considerada no programa de melhoramento genético e na indicação de cultivares (Prado et al., 2001).

Segundo Ramalho et al. (1993), quando o comportamento de duas cultivares são concordantes em dois ambientes distintos, a interação é chamada de interação simples, não acarretando maiores problemas. Entretanto, quando as cultivares possuem comportamento diverso, a interação é denominada complexa. Considerando um número maior de ambientes e de cultivares, a presença de interação complexa quase sempre indica a existência de cultivares especificamente adaptadas a ambientes particulares, bem como de outras com adaptação mais ampla, porém sem alto potencial produtivo. Em um programa de melhoramento, a avaliação de genótipos visando à identificação e recomendação de materiais superiores em diferentes ambientes é considerada por muitos autores como uma das etapas mais importante, trabalhosa e onerosa (Silva e Duarte, 2006; Maia et al., 2006; Rocha et al., 2005; Nunes et al., 2002; Prado et al., 2001; Atroch et al., 2000; Farias et al., 1997). Isso

$\overline{\text { Author for correspondence: aristotelescapone@hotmail.com }}$ 
porque exige a condução de experimentos precisos e em uma grande amplitude de condições ambientais. Existem disponíveis na literatura vários métodos para estudo e quantificação da interação genótipo $\mathrm{x}$ ambiente: $\mathrm{O}$ método tradicional, (Plaisted e Peterson, 1959; Finlay e Wilkinson, 1963; Wricke, 1965; Eberhart e Russell, 1966; Perkins e Jinks, 1968; Freeman e Perkins, 1971; Taí, 1971; Verma et al., 1978; Silva e Barreto, 1986; Lin e Binns, 1988; Cruz et al., 1989; Annicchiarico, 1992). A diferença entre os métodos origina-se nos próprios conceitos de estabilidade e nos procedimentos biométricos empregados para medi-la.

Metodologias baseadas em componentes principais, embora rotineiramente utilizadas em programas de melhoramento em estudos de diversidade genética, são pouco utilizadas em estudos da interação genótipo x ambiente (Rocha et al., 2005). Neste trabalho, a metodologia baseada nos componentes principais denominada de Centróide, foi utilizada para representar a variação do desempenho dos genótipos nos ambientes em uma dispersão no plano com poucos eixos, o que permite uma análise simultânea do desempenho de um número elevado de genótipos em virtude da facilidade de interpretação dos resultados.

O objetivo deste trabalho foi avaliar, com base na produtividade, na estabilidade e na adaptabilidade, 29 genótipos de soja sendo 25 linhagens de ciclo tardio e quatro cultivares, em seis ambientes, no Estado do Mato Grosso, através dos métodos de Annicchiarico (1992) e Centróide.

\section{MATERIAL E MÉTODOS}

Foram utilizados os dados de produtividade de grãos $\left(\mathrm{kg} \mathrm{ha}^{-1}\right)$ dos ensaios finais de competição de genótipos de soja de ciclo tardio do Programa de Melhoramento Genético do Campo Experimental Bacuri e Sales Agropecuária, conduzidos em Mato Grosso, no ano agrícola de 2004/05, nas localidades de Campo Verde, Nova Brasilândia, Vera e Rondonópolis (Tabela 1). Em Rondonópolis foram conduzidos três ensaios, denominados Rondonópolis I, II e III, que corresponderam a diferentes épocas de semeadura. Foram avaliados 29 genótipos, dos quais quatro cultivares padrão (Emgopa 315; M-Soy 8914; Tucano e Uirapuru).

Tabela 1. Altitude, latitude, longitude e data de semeadura de ensaios de competição de linhagens de soja, de ciclo tardio, no Estado do Mato Grosso.

\begin{tabular}{lcccc}
\hline Ambiente & Altitude $(\mathrm{m})$ & Latitude & Longitude & Data de semeadura \\
\hline Rondonópolis I & 227 & $16^{\circ} 28^{\prime} 15^{\prime \prime} \mathrm{S}$ & $54^{\circ} 38^{\prime} 08^{\prime \prime} \mathrm{W}$ & $07 / 11 / 2004$ \\
Rondonópolis II & 227 & $16^{\circ} 28^{\prime} 15^{\prime \prime} \mathrm{S}$ & $54^{\circ} 38^{\prime} 08^{\prime \prime} \mathrm{W}$ & $29 / 11 / 2004$ \\
Rondonópolis III & 227 & $16^{\circ} 28^{\prime} 15^{\prime \prime} \mathrm{S}$ & $54^{\circ} 38^{\prime} 08^{\prime \prime} \mathrm{W}$ & $19 / 12 / 2004$ \\
Nova Brasilândia & 540 & $14^{\circ} 57^{\prime} 25^{\prime \prime} \mathrm{S}$ & $54^{\circ} 57^{\prime} 56^{\prime \prime} \mathrm{W}$ & $04 / 12 / 2004$ \\
Campo Verde & 736 & $15^{\circ} 32^{\prime} 48^{\prime \prime} \mathrm{S}$ & $55^{\circ} 10^{\prime} 08^{\prime \prime} \mathrm{W}$ & $14 / 12 / 2004$ \\
Vera & 383 & $12^{\circ} 18^{\prime} 21^{\prime \prime} \mathrm{S}$ & $55^{\circ} 19^{\prime} 01^{\prime \prime} \mathrm{W}$ & $17 / 11 / 2004$ \\
\hline
\end{tabular}

Os experimentos foram instalados no delineamento em blocos completos casualizados, com quatro repetições. As parcelas foram formadas por quatro fileiras de plantas $(5 \mathrm{~m})$, espaçadas em $0,45 \mathrm{~m}$ entre as fileiras. A área útil da parcela foi de $3,6 \mathrm{~m}^{2}$, sendo colhidas as duas fileiras centrais, desprezando $0,5 \mathrm{~m}$ de bordadura nas extremidades.

Foram realizadas análises de variância individuais, seguindo-se uma análise de variância conjunta. A fim de implementar tais análises, utilizou-se o aplicativo computacional em genética e estatística - GENES (Cruz, 2001). Na análise conjunta, avaliou-se primeiramente a homogeneidade das variâncias residuais dos experimentos (QMR), verificada pela razão entre o maior e menor quadrado médio residual dos ensaios $(4,1252)$. Segundo Pimentel-Gomes (1990), as variâncias são consideradas homogêneas quando a relação entre o maior e o menor QMR é $\leq 7,0$.

A análise de adaptabilidade e estabilidade fenotípica dos genótipos foi feita pelos seguintes métodos: Annicchiarico (1992) e Centróide.

O método de Annicchiarico (1992) baseia-se no chamado índice de confiança genotípico, estimado por: $\quad W_{i(g)}=\hat{\mu}_{i(g)}-Z_{(1-\alpha)} \hat{\sigma}_{Z_{1(g)}}, \quad$ considerando-se todos os ambientes, em que $\hat{\mu}_{i(g)}$ é a média 
percentual dos genótipos i; $\hat{\sigma}_{Z_{1(g)}}$ é o desvio padrão dos valores de $Z_{\mathrm{ij}}$, associado ao i-ésimo genótipo; $Z_{(1-\alpha)}$ é o percentil da função de distribuição normal padrão. O coeficiente de confiança adotado foi de $75 \%$, ou seja, $\alpha=0,25$.

O método centróide, segundo Rocha et al. (2005), baseia-se na comparação de valores da distância cartesiana entre os genótipos e quatro referências ideais (ideótipos), criados com base nos dados experimentais para representar os genótipos de máxima adaptabilidade geral, máxima adaptabilidade específica a ambientes favoráveis ou desfavoráveis e os genótipos de mínima adaptabilidade. $\mathrm{O}$ ideótipo de máxima adaptabilidade geral é aquele que apresenta os valores máximos observados para todos os ambientes estudados (ideótipo I). Os ideótipos de máxima adaptabilidade específica são aqueles que apresentam máxima resposta em ambientes favoráveis e mínima resposta em ambientes desfavoráveis (ideótipo II) ou máxima resposta em ambientes desfavoráveis e mínima em ambientes favoráveis (ideótipo III). O ideótipo de mínima adaptabilidade é aquele que apresenta os menores valores em todos os ambientes estudados (ideótipo IV). Para utilização desse método, os ambientes foram classificados em favoráveis e desfavoráveis utilizando o índice ambiental como proposto por Finlay e Wilkinson, (1963).

$$
I_{j}=\frac{1}{g} \sum_{i} Y_{i j}-\frac{1}{a g} Y .
$$

Em que: $\mathrm{Y}_{\mathrm{ij}}$ : média do genótipo i, no ambiente $\mathrm{j}$; $Y .$. : total das observações; a: número de ambientes; g: número de genótipos.

Após a classificação dos ambientes, foram criados pontos referenciais, os ideótipos de resposta diferenciada a ambientes favoráveis e desfavoráveis, visando à classificação dos outros pontos do gráfico considerando os valores de distância cartesiana entre os pontos a cada um dos quatro ideótipos. Uma medida de probabilidade espacial pode ser calculada utilizando o inverso da distância entre um tratamento aos quatro ideótipos:

$$
P_{d(i, j)}=\frac{\left[\frac{1}{d i}\right]}{\sum_{i=1}^{4} \frac{1}{d i}}
$$

Em que: $P_{d(i, j)}=$ probabilidade de apresentar padrão de estabilidade semelhante ao j-ésimo centróide; $d i=$ distância do i-ésimo ponto ao jésimo centróide.

\section{RESULTADOS E DISCUSSÃO}

Os coeficientes de variação experimental variaram de 8,03 a 14,26\% (Tabela 2), indicando precisão no controle das causas de variação de ordem sistemática dos ambientes experimentais, para a produtividade de grãos, que é uma característica quantitativa muito influenciada pelo ambiente. Segundo Carvalho et al. (2003), o coeficiente de variação máximo para produtividade de grãos de soja a campo é de $16 \%$.

Tabela 2. Produtividade média de grãos $\left(\bar{Y}_{. j}\right)$, variância residual (QMR) e coeficiente de variação (CV) de ensaios de competição de linhagens de soja, de ciclo tardio, no Estado do Mato Grosso.

\begin{tabular}{lccc}
\hline \multicolumn{1}{c}{ Ambiente } & $\bar{Y}_{. j}$ & QMR & CV (\%) \\
\hline Rondonópolis I & 3122,4 & 106432,6344 & 10,45 \\
Rondonópolis II & 3155,8 & 136261,9230 & 11,70 \\
Rondonópolis III & 3053,9 & 115878,7830 & 11,15 \\
Nova Brasilandia & 3745,9 & 285335,2684 & 14,26 \\
Campo Verde & 3401,1 & 229429,6182 & 14,08 \\
Vera & 3274,9 & 69168,4747 & 8,03 \\
\hline
\end{tabular}

Os efeitos da interação $\mathrm{G} \times \mathrm{A}$ apresentaram significância a $1 \%$ de probabilidade pelo teste $\mathrm{F}$ (Tabela 3). Todos os pares de ambientes apresentaram interação do tipo complexa (Tabela 4), ou seja, houve inconsistência na superioridade do genótipo com a variação ambiental, o que dificulta a indicação das cultivares e linhagens (Cruz e Castoldi, 1991; Vencovsky e Barriga, 1992), pois não se pode, nessas circunstâncias, fazer uma recomendação uniforme para todos os locais, sem prejuízo considerável na produção obtida, relativamente à produção possível. 
Tabela 3. Análise conjunta de variância da produtividade de grãos $\left(\mathrm{kg} \mathrm{ha}^{-1}\right)$, de 29 genótipos de soja avaliados em seis ambientes, no Estado do Mato Grosso.

\begin{tabular}{lrrrrc}
\hline Fonte de variação & \multicolumn{1}{c}{ GL } & \multicolumn{1}{c}{ Soma de quadrados } & Quadrado médio & \multicolumn{1}{c}{ F } & Pr>F \\
\hline Blocos/ambientes & 18 & 6578080,2844 & 365448,9046 & - & - \\
Ambientes (A) & 5 & 37381729,6681 & 7476345,9336 & 20,457 & $<0,000$ \\
Genótipos (G) & 28 & 30873358,6178 & 1102619,9506 & 2,546 & $<0,000$ \\
Interação GxA & 140 & 60621780,6235 & 433012,7187 & 2,756 & $<0,000$ \\
Erro médio & 504 & 214625512,1594 & 157084,4503 & - & - \\
\hline
\end{tabular}

Tabela 4. Pares de ambientes, correlação entre ambientes e porcentagem da parte complexa resultante da decomposição da interação entre genótipos e pares de ambiente, segundo metodologia de Cruz e Castoldi (1991), nos ensaios de competição de linhagens de soja, no Estado do Mato Grosso.

\begin{tabular}{lcc}
\hline \multicolumn{1}{c}{ Pares de ambientes } & Correlação & Parte complexa da interação \\
\hline Rondonópolis I e Rondonópolis II & $-0,1605$ & 92,986 \\
Rondonópolis I e Rondonópolis III & 0,1345 & 72,117 \\
Rondonópolis I e Nova Brasilândia & $-0,0715$ & 81,903 \\
Rondonópolis I e Campo Verde & $-0,1964$ & 76,519 \\
Rondonópolis I e Vera & $-0,0122$ & 89,976 \\
Rondonópolis II e Rondonópolis III & $0,4026^{*}$ & 76,688 \\
Rondonópolis II e Nova Brasilândia & 0,2621 & 84,816 \\
Rondonópolis II e Campo Verde & 0,1389 & 85,668 \\
Rondonópolis II e Vera & 0,1002 & 94,166 \\
Rondonópolis III e Nova Brasilândia & 0,3207 & 82,316 \\
Rondonópolis III e Campo Verde & 0,2216 & 83,974 \\
Rondonópolis III e Vera & 0,3604 & 77,295 \\
Nova Brasilândia e Campo Verde & 0,3008 & 80,317 \\
Nova Brasilândia e Vera & 0,3173 & 78,956 \\
Campo Verde e Vera & $0,5351^{* *}$ & 53,952 \\
\hline
\end{tabular}
**, *: Significativo a 1 e $5 \%$ de probabilidade, pelo teste t.

A produtividade média de grãos variou de $3054 \mathrm{~kg}$ $\mathrm{ha}^{-1}$ (Rondonópolis III) a $3746 \mathrm{~kg} \mathrm{ha}^{-1}$ (Nova Brasilândia), com média geral entre os ambientes de $3292 \mathrm{~kg} \mathrm{ha}^{-1}$ (Tabela 5). A maior produtividade observada foi obtida pela linhagem SL 818 (4333 $\mathrm{kg} \mathrm{ha}{ }^{-1}$ ), entretanto, a maior média em todos os ambientes foi obtida pela linhagem SL 1831(3776 $\left.\mathrm{kg} \mathrm{ha}^{-1}\right)$. A menor produtividade isolada foi obtida pela linhagem SL $216\left(2211 \mathrm{~kg} \mathrm{ha}^{-1}\right)$, no entanto, a menor produtividade média em todos os ambientes foi obtida pela cultivar padrão Tucano $(2895 \mathrm{~kg}$ $\left.\mathrm{ha}^{-1}\right)$.

Tabela 5. Médias de produtividade de grãos de genótipos de soja de ciclo tardio em seis ambientes, em Mato Grosso*.

\begin{tabular}{|c|c|c|c|c|c|c|c|}
\hline \multirow{2}{*}{ Genótipos } & \multicolumn{6}{|c|}{ Ambientes } & \multirow{2}{*}{ Média } \\
\hline & RON. I & RON. II & RON. III & N. BRAS. & C. VERDE & VERA & \\
\hline SL 1 & 2894 & 3240 & 2897 & 3339 & 2783 & 3092 & 3041 \\
\hline SL 6 & 2974 & 2927 & 3266 & 3032 & 3458 & 2888 & 3091 \\
\hline SL 33 & 3165 & 2534 & 3150 & 3728 & 3694 & 3797 & 3345 \\
\hline SL 64 & 3030 & 2957 & 2384 & 4088 & 2999 & 3182 & 3107 \\
\hline SL 112 & 3452 & 2816 & 3116 & 3065 & 3575 & 3482 & 3251 \\
\hline SL 213 & 3371 & 2947 & 3117 & 3287 & 2683 & 2971 & 3062 \\
\hline
\end{tabular}




\begin{tabular}{|c|c|c|c|c|c|c|c|}
\hline SL 216 & 2949 & 3080 & 2211 & 3338 & 3684 & 3416 & 3113 \\
\hline SL 221 & 3077 & 2958 & 3304 & 3841 & 2945 & 3726 & 3309 \\
\hline SL 310 & 3100 & 3073 & 3253 & 4008 & 3995 & 3642 & 3512 \\
\hline SL 508 & 3719 & 3009 & 2981 & 4096 & 2515 & 2669 & 3165 \\
\hline SL 609 & 2929 & 3038 & 2777 & 3589 & 4039 & 2880 & 3208 \\
\hline SL 627 & 3432 & 3110 & 3328 & 4075 & 3946 & 3606 & 3583 \\
\hline SL 628 & 3143 & 3233 & 3644 & 4084 & 2924 & 3158 & 3364 \\
\hline SL 630 & 3241 & 2722 & 3046 & 3485 & 4201 & 3402 & 3350 \\
\hline SL 817 & 3075 & 3470 & 2902 & 3952 & 3405 & 3327 & 3355 \\
\hline SL 818 & 2999 & 2562 & 2719 & 4333 & 3720 & 3477 & 3302 \\
\hline SL 915 & 3133 & 3266 & 2714 & 2934 & 2502 & 2917 & 2911 \\
\hline SL 918 & 3096 & 3136 & 2898 & 4128 & 3623 & 3384 & 3377 \\
\hline SL 922 & 3151 & 3207 & 2603 & 3747 & 2977 & 3091 & 3129 \\
\hline SL 1683 & 2957 & 3005 & 3346 & 3984 & 3431 & 2955 & 3280 \\
\hline SL 1831 & 3311 & 3861 & 3576 & 3977 & 4134 & 3796 & 3776 \\
\hline SL 1923 & 3124 & 3783 & 3841 & 4220 & 3753 & 3447 & 3695 \\
\hline SL 2145 & 2886 & 3460 & 3144 & 4194 & 3622 & 3196 & 3417 \\
\hline SL 2253 & 3008 & 3863 & 2951 & 3866 & 4093 & 3150 & 3488 \\
\hline SL 2280 & 2952 & 3557 & 3607 & 3855 & 3551 & 3575 & 3516 \\
\hline EMGOPA 315 & 3088 & 3426 & 3240 & 3685 & 2881 & 3115 & 3239 \\
\hline M-SOY 8914 & 2956 & 2943 & 2765 & 3829 & 3346 & 3002 & 3140 \\
\hline TUCANO & 3269 & 2847 & 2502 & 3016 & 2771 & 2967 & 2895 \\
\hline UIRAPURU & 3072 & 3490 & 3284 & 3858 & 3384 & 3664 & 3459 \\
\hline Média & 3122 & 3156 & 3054 & 3746 & 3401 & 3275 & 3292 \\
\hline
\end{tabular}

* DMS Tukey a 5\% de probabilidade entre ambientes $=798,623 \mathrm{~kg} \mathrm{ha}^{-1} \mathrm{e}$ entre genótipos $=1045,522 \mathrm{~kg} \mathrm{ha}{ }^{-1}$. C.V. $(\%)=12,08$.

As estimativas das médias dos genótipos e parâmetros de adaptabilidade e estabilidade das cultivares e linhagens obtidas pelos métodos de Annicchiarico (1992) e Centróide (Rocha et al., 2005), encontram-se na Tabela 6.

No método proposto por Annicchiarico, a estabilidade é medida pela superioridade do genótipo em relação à média de cada ambiente. $\mathrm{O}$ método baseia-se na estimação de um índice de confiança de um determinado genótipo mostrar comportamento relativamente superior.

Com base no índice de confiança ou de recomendação, pode-se indicar as linhagens: SL 1831, SL 1923, SL 627, SL 2280, SL 310, SL 2253, SL 2145, SL 918 e SL 817, além da cultivar Uirapuru, considerando a sua produtividade em todos os ambientes, ou seja, adaptabilidade geral. Nos ambientes classificados como favoráveis
(Nova Brasilândia e Campo Verde) as linhagens SL 1831, SL 1923, SL 627, SL 2280, SL 310, SL 2253, SL 2145, SL 918, SL 817, SL 630, SL 33, SL 1683, SL 818 e SL 609, além da cultivar Uirapuru, apresentaram comportamento mais estável e com probabilidade de apresentar produtividade superior a média de cada ambiente. Nos ambientes classificados como desfavoráveis (Rondonópolis I, II, II e Vera) destacaram-se as linhagens: SL 1831, SL 1923, SL 627, SL 2280, SL 310, SL 628 e SL 221, alem da cultivar Uirapuru.

O conceito de adaptabilidade e estabilidade utilizado no método centróide diferencia dos demais, uma vez que o genótipo de máxima adaptação específica não é aquele que apresenta bom desempenho nos grupos de ambientes favoráveis ou desfavoráveis, mas sim o genótipo 
que apresenta valores máximos para determinado mínimo para o outro conjunto (Rocha et al., 2005). grupo de ambientes (favoráveis e desfavoráveis) e

Tabela 6. Produtividade média $\left(\mathrm{kg} \mathrm{ha}^{-1}\right)$, Índice de confiança $W_{i}$ (geral, favorável e desfavorável) e a classificação dos genótipos em um dos quatro grupos caracterizados pelos centróides e a probabilidade associada a sua classificação dos genótipos de soja de ciclo tardio, em Mato Grosso.

\begin{tabular}{|c|c|c|c|c|c|c|c|c|c|}
\hline \multirow[b]{2}{*}{ Genótipos } & \multirow[b]{2}{*}{ Média } & \multicolumn{3}{|c|}{ Annicchiarico (1992) ${ }^{1 /}$} & \multicolumn{5}{|c|}{ Centróide $^{21}$} \\
\hline & & $\begin{array}{c}W_{i} \\
\text { Geral }\end{array}$ & $W_{i}$ fav. & $W_{i}$ desf. & Grupo & $\mathrm{P}(\mathrm{I})$ & $\mathrm{P}$ (II) & $\begin{array}{c}\mathrm{P} \\
\text { (III) }\end{array}$ & $\mathrm{P}(\mathrm{IV})$ \\
\hline SL 1831 & 3776 & 112,87 & 110,88 & 113,48 & $\mathrm{I}$ & 0,564 & 0,150 & 0,171 & 0,115 \\
\hline SL 1923 & 3695 & 109,75 & 111,05 & 109,43 & I & 0,477 & 0,176 & 0,207 & 0,140 \\
\hline SL 627 & 3583 & 107,17 & 111,00 & 105,35 & $\mathrm{I}$ & 0,395 & 0,242 & 0,197 & 0,166 \\
\hline SL 2280 & 3516 & 104,71 & 103,36 & 105,86 & $\mathrm{I}$ & 0,358 & 0.203 & 0.260 & 0.179 \\
\hline SL 310 & 3512 & 104,42 & 110,21 & 101,82 & I & 0,350 & 0.272 & 0.198 & 0.180 \\
\hline SL 2253 & 3488 & 102,47 & 108,45 & 99,33 & I & 0,331 & 0.276 & 0.204 & 0.189 \\
\hline UIRAPURU & 3459 & 103,58 & 100,56 & 105,43 & I & 0,320 & 0.214 & 0.269 & 0.197 \\
\hline SL 2145 & 3417 & 101,46 & 108,16 & 98,62 & I & 0,307 & 0.287 & 0.207 & 0.200 \\
\hline SL 918 & 3377 & 100,72 & 107,64 & 98,23 & II & 0,276 & 0.318 & 0.196 & 0.210 \\
\hline SL 628 & 3364 & 99,22 & 93,04 & 101,97 & III & 0,275 & 0,218 & 0,284 & 0,223 \\
\hline SL 817 & 3355 & 100,33 & 101,75 & 99,52 & I & 0,276 & 0.268 & 0.230 & 0.226 \\
\hline SL 630 & 3350 & 98,24 & 102,37 & 96,14 & II & 0,266 & 0,319 & 0,198 & 0,217 \\
\hline SL 33 & 3345 & 98,21 & 102,31 & 96,14 & II & 0,267 & 0,281 & 0,222 & 0,230 \\
\hline SL 221 & 3309 & 97,87 & 91,48 & 101,08 & III & 0,250 & 0,219 & 0,287 & 0,243 \\
\hline SL 818 & 3302 & 95,99 & 111,31 & 90,21 & II & 0,223 & 0,404 & 0,164 & 0,208 \\
\hline SL 1683 & 3280 & 97,45 & 102,55 & 95,13 & II & 0,257 & 0,292 & 0,216 & 0,235 \\
\hline SL 112 & 3251 & 96,12 & 88,96 & 99,51 & III & 0,236 & 0,233 & 0,267 & 0,263 \\
\hline EMGOPA & & & & & & & & & \\
\hline 315 & 3239 & 96,30 & 88,90 & 100,46 & III & 0,230 & 0,211 & 0,299 & 0,260 \\
\hline SL 609 & 3208 & 94,24 & 102,84 & 91,24 & II & 0,215 & 0,385 & 0,171 & 0,229 \\
\hline SL 508 & 3165 & 91,54 & 84,79 & 94,14 & IV & 0,212 & 0,240 & 0,249 & 0,298 \\
\hline M-SOY & 3140 & 93,91 & 99,54 & 92,04 & II & 0,200 & 0,327 & 0,189 & 0,283 \\
\hline SL 922 & 3129 & 92,99 & 91,35 & 93,46 & IV & 0,198 & 0,259 & 0,222 & 0,322 \\
\hline SL 216 & 3113 & 90,86 & 94,99 & 88,40 & II & 0,199 & 0,314 & 0,194 & 0,294 \\
\hline SL 64 & 3107 & 91,05 & 94,61 & 89,00 & II & 0,192 & 0,312 & 0,190 & 0,306 \\
\hline SL 6 & 3091 & 91,74 & 87,29 & 93,59 & IV & 0,208 & 0,242 & 0,244 & 0,305 \\
\hline SL 213 & 3062 & 90,62 & 81,60 & 96,35 & IV & 0,179 & 0,195 & 0,278 & 0,348 \\
\hline SL 1 & 3041 & 90,71 & 84,07 & 94,94 & IV & 0,180 & 0,207 & 0,256 & 0,357 \\
\hline SL 915 & 2911 & 85,73 & 75,03 & 93,36 & IV & 0,149 & 0,172 & 0,248 & 0,431 \\
\hline TUCANO & 2895 & 85,71 & 80,80 & 89,27 & IV & 0,131 & 0,175 & 0,184 & 0,509 \\
\hline
\end{tabular}

${ }^{1 /}$ Alfa $=0,25 ; \mathrm{Z}(1$-alfa $)=0,2734 ;{ }^{2 /}$ Em que: Ideótipo I = Adaptabilidade geral (++); Ideótipo II $=$ Adaptabilidade especifica a ambientes favoráveis (+-); Ideótipo III = Adaptabilidade específica a ambientes desfavoráveis (-+); Ideótipo IV = Pouco adaptado (--).

Após a classificação dos ambientes, os ideótipos estimados com base nos dados originais foram acrescidos na análise (Tabela 7). Uma vez estabelecidos os valores médios de cada ideótipo utilizou-se a análise de componentes principais envolvendo os 29 genótipos iniciais e quatro outros representativos, que na análise gráfica, representam os quatro centróides em torno dos quais foi avaliada a dispersão dos demais. A obtenção dos autovalores via metodologia dos componentes principais, partindo dos dados originais incluídos os ideótipos, mostram que apenas dois componentes principais são suficientes para explicar proporções superiores a $67 \%$ da variação total (Tabela 8). Uma vez constatada a suficiência de dois autovalores na representação da 
variação total, a avaliação da posição dos bidimensionais (Gráfico 1).

genótipos pode ser feita por meio de gráficos

Tabela 7. Classificação dos ambientes utilizando o índice ambiental e estabelecimento dos ideótipos, calculados pelo método CENTRÓIDE, dos genótipos de soja de ciclo tardio, na safra 2004/2005 em Mato Grosso

\begin{tabular}{lcccccccc}
\hline Ambientes & Média & Ij & Máximo & Mínimo & $\begin{array}{c}\text { Ideótipo } \\
\text { I }\end{array}$ & $\begin{array}{c}\text { Ideótipo } \\
\text { II }\end{array}$ & $\begin{array}{c}\text { Ideótipo } \\
\text { III }\end{array}$ & $\begin{array}{c}\text { Ideótipo } \\
\text { IV }\end{array}$ \\
\hline Rond. I & 3122 & $-169,97$ & 3719 & 2886 & 3719 & 2886 & 3719 & 2886 \\
Rond. II & 3156 & $-136,54$ & 3863 & 2534 & 3863 & 2534 & 3863 & 2534 \\
Rond. III & 3054 & $-238,41$ & 3841 & 2211 & 3841 & 2211 & 3841 & 2211 \\
N. Brás. & 3746 & 453,58 & 4333 & 2934 & 4333 & 4333 & 2934 & 2934 \\
C. Verde & 3401 & 108,78 & 4201 & 2502 & 4201 & 4201 & 2502 & 2502 \\
Vera & 3275 & $-17,42$ & 3797 & 2669 & 3797 & 2669 & 3797 & 2669 \\
\hline
\end{tabular}

Em que: Ideótipo I = Adaptabilidade geral (++); Ideótipo II = Adaptabilidade especifica a ambientes favoráveis (+); Ideótipo III = Adaptabilidade específica a ambientes desfavoráveis (-+); Ideótipo IV = Pouco adaptado (--).

Tabela 8. Estimativa dos autovalores obtidos conforme a técnica de componentes principais e fração cumulativa da variância explicada por estes

\begin{tabular}{ccc}
\hline Raiz & Raiz (\%) & \% Acumulada \\
\hline 2,52009 & 42,00152 & 42,00152 \\
1,50599 & 25,09991 & 67,10144 \\
0,70239 & 11,70657 & 78,80802 \\
0,60079 & 10,01328 & 88,82131 \\
0,37914 & 6,31913 & 95,14045 \\
0,29157 & 4,85955 & 100,0 \\
\hline
\end{tabular}

I - Adap. geral; II - Adap. esp. favorável; III - Adap. esp. desfavorável e IV - Pouco

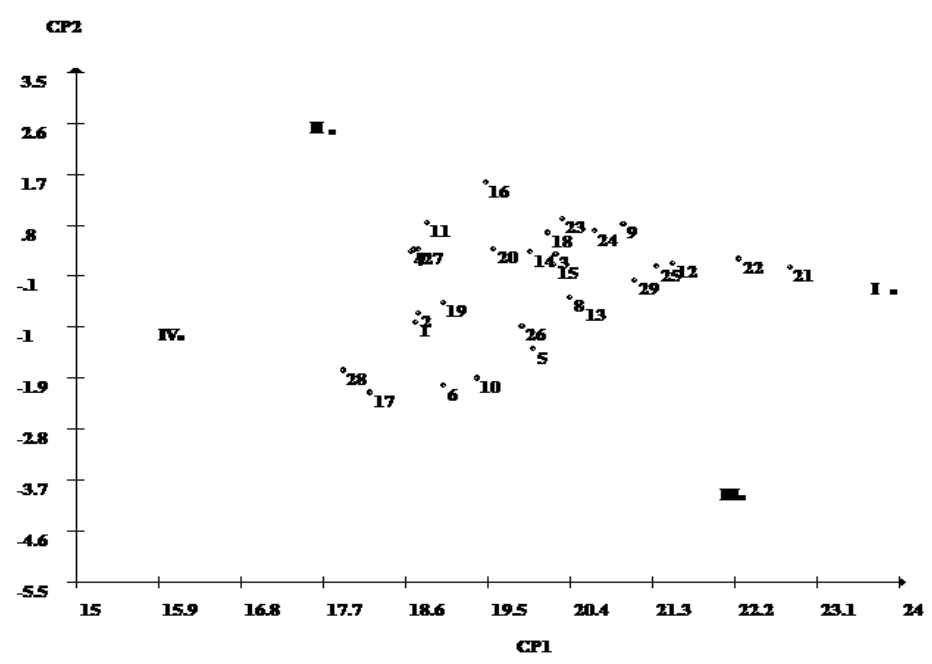

Gráfico 1 - Dispersão gráfica dos escores em relação aos dois primeiros componentes principais obtidos da análise da produtividade de 29 genótipos de soja de ciclo tardio avaliados em seis ambientes. Os quatro pontos numerados com algarismos romanos representam os centróides. Genótipos: 1 - SL 1; 2 - SL 6; 3 - SL 33; 4 - SL 64; 5 - SL 112;6 - SL 213; 7 - SL 216;8 - SL 221; 9 - SL 310; 10 - SL 508; 11 - SL 609; 12 - SL 627; 13 - SL 628; 14 - SL 630; 15 - SL 817; 16 - SL 818; 17 - SL 915; 18 - SL 918; 19 - SL 922; 20 - SL

A análise visual de gráficos de componentes principais permite avaliar que os genótipos apresentam distribuição heterogênea para a produtividade de grãos e que existem pontos de 
maior proximidade a todos os quatro centróides, possibilitando uma recomendação de genótipos de adaptabilidade geral ou recomendação de genótipos de adaptabilidade específica a um subgrupo de ambientes (Carvalho et al., 2002). As linhagens 21 (SL 1831) e 22 (SL 1923 foram classificadas como sendo de adaptabilidade geral por localizarem-se mais próximos do ideótipo I. Entretanto, a maioria dos pontos (genótipos) foram plotados na região central do gráfico, dificultando a classificação. Neste caso, utilizou-se o inverso do valor da distância entre um ponto aos quatro centróides como estimativa da confiabilidade de agrupamento dos genótipos (Rocha et al., 2005). Dessa maneira, um ponto equiidistante aos quatro pontos referenciais apresenta valores de probabilidade de $25 \%$ de pertencer a qualquer um dos grupos e, portanto, quanto mais o valor de probabilidade diferir de $25 \%$, maior será a certeza em concluir o agrupamento do genótipo. Segundo Rocha et al., (2005), valores de probabilidade próximos ou superiores a 50\% indicam boa confiabilidade no agrupamento.

Na Tabela 6 são apresentadas às classificações dos genótipos a um dos quatro grupos $\mathrm{e}$ a probabilidade associada as suas classificações. Pelo método Centróide as linhagens SL 1831, SL 1923, SL 627, SL 2280, SL 310, SL 2253, SL 2145 e SL 817 além da cultivar Uirapuru, foram classificadas com sendo de ampla adaptabilidade e estabilidade, destacando-se as linhagens SL 1831 e SL 1923. Para condições específicas de ambientes desfavoráveis, foram classificadas as linhagens SL 918, SL 630, SL 33, SL 818, SL 1683, SL 609, SL 216 e SL 64, além da cultivar M-Soy 8914. Já para condições específicas de ambientes desfavoráveis, foram classificadas por essa metodologia as linhagens SL 628, SL 221 e SL 112 e a cultivar Emgopa 315.

Observa-se no gráfico de dispersão (Gráfico 1) e na Tabela 6 uma tendência de aumento na média de produtividade dos genótipos de soja à medida que estes se aproximam do centróide I (adaptabilidade geral). De acordo com Rocha et al., (2005), quanto menor for a diferença entre um genótipo qualquer e o ideótipo I, menor será a diferença entre este e o genótipo de máximo desempenho em todos os ambientes, fazendo com que a adaptabilidade geral esteja necessariamente associada ao melhor desempenho.

Comparando a classificação da estabilidade fenotípica dos genótipos obtida pelo método
Centróide com classificação obtida pela metodologia proposta por Annicchiarico (1992), observa-se que houve coincidência na classificação dos genótipos para amplas condições ambientes (alta estabilidade e adaptabilidade geral) e para condições especificas de ambientes favoráveis e desfavoráveis.

\section{CONCLUSÕES}

Houve coerência na classificação da adaptabilidade e estabilidade dos genótipos pelas metodologias analisadas;

As linhagens de soja SL 1831, SL 627, SL 2280 e SL 310 apresentaram melhor comportamento para amplas condições ambientais e a linhagem SL 818 para condições específicas de ambientes favoráveis, tendo em vista a produtividade de grãos.

\section{AGRADECIMENTOS}

Ao Conselho Nacional de Desenvolvimento Científico e Tecnológico - CNPq pelo apoio financeiro em forma de bolsa (Doutorado) e as Empresas Bacuri/Soygene e Sales Agropecuária pela condução dos ensaios de campo.

\section{RESUMO}

Este trabalho teve o objetivo de avaliar a produtividade, a estabilidade e a adaptabilidade de 29 genótipos de soja [Glycine $\max (\mathrm{L}$.) Merrill], em seis ambientes no Estado do Mato Grosso. O delineamento experimental foi blocos ao acaso, com quatro repetições. Os ensaios foram conduzidos no ano agrícola de 2004/05 nos municípios de Rondonópolis, Campo Verde, Nova Brasilândia e Vera. Para avaliação da adaptabilidade e estabilidade, utilizou-se os métodos de Annicchiarico (1992) e Centróide. O rendimento médio de grãos foi de $3054 \mathrm{~kg} \mathrm{ha}^{-1}$ (Rondonópolis III) a $3746 \mathrm{~kg} \mathrm{ha}^{-1}$ (Nova Brasilândia), com média geral entre os ambientes de $3292 \mathrm{~kg} \mathrm{ha}{ }^{-1}$. Com base nas metodologias de Annicchiarico e Centróide, as linhagens SL 1831, SL 1923, SL 627, SL 2280 e SL 310 foram classificadas como as mais produtivas, adaptadas e estáveis, havendo, portanto, coerência entre tais metodologias. Foram recomendadas as linhagens de soja SL 1831, SL 627, SL 2280 e SL 310 para amplas condições ambientais e a linhagem SL 818 para condições específicas de ambientes favoráveis, tendo em vista a produtividade de grãos.

Palavras-chave: Glycine max, Produtividade, estabilidade, adaptabilidade, uni-multivariada 


\section{REFERÊNCIAS}

Annicchiarico, P. (1992), Cultivar adaptation and recommendation from alfafa trials in Northern Italy. Journal of Genetics and Breeding, 46, 269278.

Atroch, A. L.; Soares, A. A.; Ramalho, M. A. P. (2000), Adaptabilidade e estabilidade de linhagens de arroz de sequeiro testadas no Estado de Minas Gerais. Ciência e Agrotecnologia, 24, 541-548.

Carvalho, C. G. P.; Arias, C. A. A.; Toledo, J. F. F.; Almeida, L. A.; Kiihl, R. A. S.; Oliveira, M. F.; Hiromoto, D. M.; Takeda, C. (2003), Proposta de classificação dos coeficientes de variação em relação à produtividade e altura da planta de soja. Pesquisa Agropecuária Brasileira, 38, 187-193.

Carvalho, H. W. L.; Silva, M. L.; Cardoso, M. J.; Santos, M. X.; Tabosa, J. N.; Carvalho, C. L.; Lira, M. A. (2002), Adaptabilidade e estabilidade de cultivares de milho no Nordeste brasileiro no triênio de 1998 a 2000. Pesquisa Agropecuária Brasileira, 37, 1581-1588.

Cruz, C. D. (2001), Programa GENES - aplicativo computacional em genética e estatística, Viçosa: UFV, Imprensa Universitária. 542p.

Cruz, C. D. and Castoldi, F. L. (1991), Decomposição da interação genótipos ambientes em partes simples e complexa. Revista Ceres, 38, 422-430.

Cruz, C. D.; Torres, R. A.; Vencovsky, R. (1989), An alternative approach to the stability analysis proposed by Silva and Barreto. Revista Brasileira de Genética, 12, 567-580.

Eberhart, S. A. and RusselL, W. A. (1966), Stability parameters for comparing varieties. Crop Science, 6, 36-40.

Farias, F. J. C.; Ramalho, M. A. P. R.; Carvalho, L. P.; Moreira, J. A. N.; Costa, J. N. (1997), Parâmetros de estabilidade propostos por Lin \& Binns (1988) comparados com o método da regressão. Pesquisa Agropecuária Brasileira, 32, 407-414.

Finlay, K. W. and Wilkinson, G. N. (1963), The analysis of adaptation in plant-breeding programme. Australian Journal of Agricultural Research, 14, 742-754.

Freeman, G. H. and Perkins, J. M. (1971), Environmental and genotype-environmental components of variability. VIII. Relations between genotypes grown in different environments and measures of these environments. Heredity, 27, 1523.

Lin, C. S. and Binns, M. R. (1988), A superiority measure of cultivar performance for cultivars $\mathrm{x}$ location data. Canadian Journal of Plant Science, 68, 193-198.

Maia, M. C. C.; Vello, N. A.; Rocha, M. M.; Pinheiro, J. B.; Silva Junior, N. F. (2006), Adaptabilidade e estabilidade de linhagens experimentais de soja selecionadas para caracteres agronômicos através de método uni-multivariado. Bragantia, 65, 215-226.

Nunes, G. H. S.; Rezende, G. D. S. P. M.; Ramalho, M. A. P.; Santos, J. B. S. (2002), Implicações da interação genótipos $\mathrm{x}$ ambientes na seleção de clones de eucalipto. Cerne, 8, 49-58.

Perkins, J. M. e Jinks, J. L. (1968), Environmental and genotype-environmental components of variability. III. Multiple lines and crosses. Heredity, 23, 339-356.

Pimentel-Gomes, F. (1990), Curso de estatística experimental. 13Ed. Piracicaba - SP: Nobel. 468p.

Plaisted, R. L. and Peterson, L. C. (1959), A technique for evaluating the ability of selections to yield consistently in different locations or seasons. American Potato Journal, 36, 381-385.

Prado, E. E. P.; Hirimoto, D. M.; Godinho, V. P. C.; Utumi, M. M. Ramalho, A. R. (2001), Adaptabilidade e estabilidade de cultivares de soja em cinco épocas de plantio no cerrado de Rondônia. Pesquisa Agropecuária Brasileira, 36, 625-635.

Ramalho, M. P.; Santos, J. B.; Zimmermann, M. J. (1993), Interação dos genótipos por ambientes. In: Genética quantitativa em plantas autógamas: aplicações ao melhoramento do feijoeiro. UFG, Goiânia, 1, 137-170. 
Rocha, R. B.; Muro-Abad, J. I.; Araujo, E. F.; Cruz, C. D. (2005), Avaliação do método centróide para estudo de adaptabilidade ao ambiente de clones de Eucalyptus grandis. Ciência Florestal, 15, 255-266.

Silva, W. C. J. and Duarte, J. B. (2006), Métodos estatísticos para estudo de adaptabilidade e estabilidade fenotipica em soja. Pesquisa Agropecuária Brasileira, 41, 23-30.

Silva, J. G. C. and Barreto, J. N. (1986), An application of segmented linear regression to the study of genotype $\mathrm{x}$ environment interaction. Biometrics, 41, 1093-1093.
Tai, G. C. C. 1971. Genotype stability analysis and its a application to potato regional trials. Crop Science, 11, 184-190.

Verma, M. M.; Chahal, G. S.; Murty, B. R. (1978), Limitations of conventional regression analysis: a proposed modification. Theoretical and Applied Genetics, 53, 89-91.

Vencovsky, R. and Barriga, P. (1992), Genética biométrica no fitomelhoramento. Ribeirão PretoSP: Sociedade Brasileira de Genética. 486p.

Wricke, G. (1965), Zur Berechnung der Ökovalenz bei Sommerweizen und Hafer. Zeitschrift für Pflanzenzüchtung, 52, 127-138. 